\title{
Themes from Ontology, Mind, and Logic
}

\author{
Present and Past \\ Essays in Honour of Peter Simons
}

Edited by

Sandra Lapointe

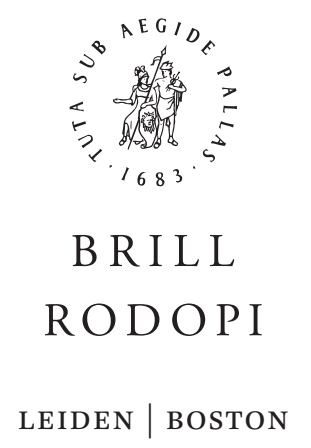

For use by the Author only | (C) 2015 Koninklijke Brill NV 


\section{TABLE OF CONTENTS}

Preface $\ldots \ldots \ldots \ldots \ldots \ldots \ldots \ldots \ldots \ldots \ldots \ldots \ldots \ldots \ldots \ldots$ vii

\section{Part I: Ontology}

Fabrice CORREIA: Logical Grounding and First-Degree Entailments

Ingvar JOHANSSON: Collections as One-and-Many-On the $\mathrm{Na}$ ture of Numbers . . . . . . . . . . . . . . . 17

Kathrin KOSLICKI: In Defense of Substance . . . . . . . . . . . 59

Uriah KRIEGEL: How to Speak of Existence: A Brentanian Approach to (Linguistic and Mental) Ontological Commitment . . . . . . . .

Maria Elisabeth REICHER: Computer-generated Music, Authorship, and Work Identity . . . . . . . . . . . . . . . . . 107

Benjamin SCHNIEDER: The Asymmetry of 'Because' . . . . . . . . . 131

Johanna SEIBT: Non-Transitive Parthood, Leveled Mereology, and the Representation of Emergent Parts of Processes . . . . . . . . . 165

Theodore SIDER: Nothing Over and Above . . . . . . . . . . . 191

David WOODRUFF SMITH: On Basic Modes of Being: Metametaphysical Reflections in Light of Whitehead, Husserl, Ingarden, Hintikka . . . . . . . . . . . . . . . .

\section{Part II: Mind}

Kevin MULLIGAN: Annehmen, Phantasieren und Entertaining. Husserl und Meinong . . . . . . . . . . . . . . . . 245

Mark TEXTOR: Meaning, Entertaining, and Phantasy Judgement . . 285

Maria VAN DER SCHAAR: The Things We Call True ........ 303 
PART III: Logic

Bob HALE \& Crispin WRIGHT: Bolzano's Definition of Analytic Propositions . . . . . . . . . . . . . . . . . 325

Wolfgang KÜNNE: On Having a Property. Corrigenda in Bolzano's Wissenschaftslehre..................... 365

Edgar MORSCHER: The Logic of Truth . . . . . . . . . . . . . . . 409

Jan WOLEŃSKI: An Analysis of Logical Determinism . . . . . . . . 423

For use by the Author only | (c) 2015 Koninklijke Brill NV 


\title{
NON-TRANSITIVE PARTHOOD, LEVELED MEREOLOGY, AND THE REPRESENTATION OF EMERGENT PARTS OF PROCESSES
}

\author{
Johanna SEIBT \\ Section for Philosophy and the History of Ideas \\ Aarhus University
}

\section{Summary}

Processes have mereological structure, just like things and stuffs, but part-whole relations for processes have not received much attention in the literature so far. As I explain in the first part of this paper, the main reason for this curious neglect is that extant classical and non-classical mereologies have hidden built-in restrictions on the type of entities that can stand in the part-relations formalized by these mereologies; processes and other non-particular individuals do not fulfill the given restrictions. In a second step I introduce a non-classical mereological system (LEM) which is free of these restrictions and operates with a nontransitive 'is-part' relation, capturing the most general sense of mereological association: 'belongs-with.' In a third step I discuss to what extent LEM can be used to formally represent our qualitative reasoning about processes.

Processes have mereological structure, just like things. Unlike things, however, processes have parts that can be said to 'emerge,' in a fairly strong sense of that term. Curiously, however, the debate about emergence, both the older debate in the 1950s and the more recent discussion since 1990, has largely been conducted with focus on emergent properties of things rather than on emergent (parts of) processes. In fact, part-whole relations for processes altogether have not received much attention in the literature so far. ${ }^{1}$ Why have we all but forgotten about processes in analytical ontol-

1. As in many other areas in ontology, Peter Simons' short discussion of the topic in his 1987 (129-147) offered a seminal pointer by creating an association between the formal mereology of stuffs and the mereology of processes, which, in informal terms and with focus on the semantics of verbal aspects, had been observed by Dowty (1977) and, in particular, Mourelatos (1978) . 
ogy, and in the debate about emergence and in mereology in particular? What would it take to devise a theory of parts for processes, and which theoretical benefits might we gain from it, besides extending the scope of ontological analysis? These questions lead into fairly unexplored terrain and the following considerations will do little more than point to some possible trailheads towards a non-Whiteheadian process ontology. If the following sketch will not succeed in recruiting fellow explorers, if it merely will serve to redirect readers towards the highway that Whitehead blasted into the jungle of metaphysics and hid under the overgrowth of foreign terminology, they will have fulfilled a dialectical function that is not entirely unwelcome. ${ }^{2}$

In the first part of this paper I question the ubiquitous belief that 'partof' in its most basic sense is a transitive relation. I suggest that there is a joint reason for the neglect of processes in mereology and for the dogma of transitivity - these are, among many others, the effects of a longstanding theoretical tradition in ontology that even the 'analytical' reformation of the discipline in the first half of the $20^{\text {th }}$ century could not shed. The theoretical presuppositions of this tradition-which I call the 'substance paradigm' or the 'myth of substance'-generate restrictions on the types of entities that can count as ontologically basic, as well as on the types of entities that are primary targets of ontological investigation. In consequence of this restriction on entity types, a certain type of part-whole relation took center-stage in the development of mereology: spatial parthood on spatial regions. The transitivity of this sort of parthood also abetted the idea that mereology and emergence are anathema, and that one cannot model emergence using mereological relationships. In a second step I sketch a non-classical mereological system (LEM: Leveled Mereology), which operates with a non-transitive 'is-part' relation and is designed to capture the logical properties of the most general and inferentially weakest part-whole relation: 'belongs-with.' In a third step I show how claims about the emergent parts of processes can be formulated with the tools of LEM. I conclude with a brief discussion of the significance of this formal presentation.

As far as I can see, I may have been the first one (cf. Seibt 1990, ch. 5) to pursue this pointer systematically and to further explore the mereology of processes alias "dynamic masses": cf. the references listed on pages 174 and 183 below.

2. C.f. e.g., Simons (2000). 


\section{Classical transitive parthood and the "myth of substance"}

The processing of part-whole relationships seems to be one of our most basic cognitive capacities. In terms of the partly phenomenological, partly logical analysis of cognition that philosophers used to call 'transcendental' and still often undertake, it would seem possible to argue, for example, that part-whole relationships are cognitively more fundamental than memberset relationships. The cognitive 'grasp' of member-set relationships, one might say, presupposes the capacity to focus on some feature of an entity and to 'represent' the entity in terms of that feature and to associate it with others selectively 'represented' by similar features. ${ }^{3}$ Traditionally, this cognitive capacity of selective representation has been called 'abstraction,' retaining the terminology, if not the theoretical model, of the Scholastic account of perception. But one might also argue that the selective representation that is prerequisite for member-set relations is connected to the linguistic phenomenon of metonymy or, more precisely, synekdoche. In synekdoche an entity $\mathrm{E}$ is referred to by using an expression $\mathrm{L}$ which denotes a part of that entity, e.g., 'I need a new set of wheels,' or: 'what we want are more bums on seats'; alternatively, $\mathrm{L}$ denotes the whole of which $\mathrm{E}$ is a part, e.g., 'Do you need to use the bathroom?' or 'I am going to fill up the car with petrol. ${ }^{\prime 4}$ Synekdoche, one might suggest, manifests at the linguistic level an underlying cognitive ability to grasp 'at a glance' a situation with different features and a basic relationship of asymmetric 'belonging with,' so that the simple or complex item on either side of this relationship can be foregrounded to refer to the other. In short, guided by the linguistic phenomenon of synekdoche one might hypothesize that member-set relationships are cognitively grounded in referential 'mechanisms' where features of a situation come to stand for the whole situation, or vice versa.

Whether the hypothesis that part-whole relationships are cognitively prior to member-set relationships can be argued for along these lines would need to be investigated in cognitive psychology or cognitive science. I have introduced it here since it draws attention to a feature of part-whole relationships that can be easily confirmed by introspection. It is phenomenologically striking and systematically important, in my view, that partwhole relations, some at least, can be grasped 'at a glance', immediately-we seem to be able to directly perceive them or at least process them without

3. In the context of this paper I want to bracket the question which, if any, notion of mental representation should still be used in present-day philosophy.

4. Cf. Rijkhoff (2015), whence also all sample sentences are taken. 
any noticeable inferential step. This phenomenologically non-inferential character of the part-whole relation holds in particular for spatial parts. Whether something is spatially part of something else we simply see-we take it in as an integral aspect of the scenery presented.

Let (A1) be the assumption that phenomenal immediacy (or non-inferentiality) is a criterion (i.e., necessary condition) for the cognitive basicness of a relationship. The above argument for the cognitive priority of parthood hinges on (A1) and the phenomenological datum of the phenomenal immediacy of spatial parthood. In contrast, membership requires more complex processing and is not phenomenally immediate; moreover, membership seems to involve the processing of spatial parthood in one of its constitutive components, that is, the referential shift manifested in synekdoche, from whole entities to features. Encouraged by this line of reasoning one might further be tempted to argue that foundational theories in analytical ontology should not resort to set-theory but use classical extensional mereology as a basic formal framework. More explicitly, one might try to establish such a recommendation on the basis of (A1), the immediacy criterion for cognitive basicness, and the following additional assumptions:

(A2) Foundational ontologies should aim to operate with formal frameworks that represent cognitively basic relationships.

(A3) Spatial parthood is a cognitively basic relationship.

(A4) Classical extensional mereology (CEM) models spatial parthood per se, i.e., spatial parthood on arbitrary types of entities.

(A5) (CEM) models the most generic notion of parthood that is cognitively basic in the sense of (A1), i.e., there is no other cognitively basic part-whole relationship that is more generic or comprehensive than spatial parthood per se and is not modelled by (CEM).

I suspect that assumption (A4) will appear straightforward to many mereologists. ${ }^{5}$ To be sure, due to Simons' pioneering 1987 there is general acknowledgement among mereologists that (CEM) cannot model all part-relations, especially those that carry modal information, such as constitutive parthood or material constitution. But modal part-whole relationships are precisely not phenomenologically immediate, and so one might hold on to the idea that (CEM) captures at least spatial parthood, the cognitively basic parthood relation. Significantly, the basic principles

5. For a clear statement of this commitment cf. e.g., Varzi 2006:1. 
of (CEM) are typically illustrated with geometric diagrams of spatial inclusion. What has gone unnoticed, however, is that is it not spatial parthood per se that provides a model for (CEM) but spatial parthood on spatial regions. Objects, stuffs, activities, events, persons, collectives etc. all have spatial parts, but these do not coincide with the items occupying regions that are spatial parts of the spatial region occupied by the object. Consider the following sentences, letting ' $S$ ' denote a certain screw that fixes a cable running inside one of the walls of my house:

[1] A screw is a spatial part of a house. (false)

[2] $S$ is a spatial part of my house. (?)

[3] The spatial region occupied by $S$ is a spatial part of the region occupied by this house. (true)

Sentence [1], which is a generic statement, is false since screws are not typically used in the construction of houses. The falsity of [1] however disinclines us to treat [2] as true, especially if we are also provided with sentence [3] as a clarification of why we initially might have considered [2] as true (what we would have meant had we accepted [2] as true). ${ }^{6}$ But if [2] is false, spatial parthood on objects such as houses is not a strictly transitive relation as required by (CEM) - the wall is a spatial part of my house but something spatially in the wall apparently is not.

Similar counterexamples to the transitivity of spatial parthood can be constructed for other kinds of objects:

[4] An influenza virus is a spatial part of a human organism. (false)

[5] (I have influenza and carry virus V.) V is a spatial part of my body. (?)

[6] The spatial region occupied by $\mathrm{V}$ is a spatial part of the region occupied by my body. (true)

The example is to show that something spatially included in the spatial part of a thing may fail to qualify as a spatial part of that thing. As illustrated

6. This is an empirical claim, as is any claim about 'what we would say' or 'would not say', i.e., about our normative dispositions. Note that temporal relativizations of the second and third sentences ("At $t, \ldots$ ".) are omitted, here and in the examples below. This and the following examples can also serve to make a methodological point in passing. I do not think that our so-called "metaphysical intuitions" are stable across contextualizations—as sociologists and psychologists are well aware of, already the order in which certain information is presented affects a subjects responses. 
by sentences [1] through [6], the 'transitivity failure' of spatial parthood on objects arises due to the fact that our nouns for objects are abbreviations for functional organizations. Objects such as houses, desks, doors, or dishwashers have functional organizations (functional structures), which reflect the dispositional features of objects relative to physical requirements and/or socio-historical contingencies. Whether something is a spatial part of an object we determine relative to the functional organization of this kind of object. In general, if we determine the spatial parts of an object $\mathrm{O}$, spatial parthood is transitive as long as it pertains to items that belong to the functional organization of $\mathrm{O}^{7}$

Problems with the transitivity of spatial parthood have been discussed since Aristotle but mostly with focus on the alleged spatial "granularity" of stuffs ('is one oxygen molecule a spatial part of water?') and the alleged temporal granularity of activities ('is my inhaling part of my singing?'). ${ }^{8}$ It is important, however, to separate the discussion of the granularity of stuffs and processes from transitivity failures for spatial parthood on stuffs and processes that arise for claims about spatial items that are not included in the functional organization of the stuff or process in question. Consider again the following triplets of sentences:

[7] Radon is a spatial part of gin-and-tonic. (false)

[8] (The tonic water I used for my drink is contaminated with certain amount of radon, call it R.) $R$ is a spatial part of my (glass of) gin-and-tonic.

[9] The (scattered) spatial region occupied by $\mathrm{R}$ is a spatial part of the region occupied by my (glass of) gin-and-tonic. (true)

[10] The pistons' rattling is a spatial part of an engine's running. (false)

[11] My car is very old and the pistons in my car's engine rattle; call that activity of rattling ' $R$ ' and the activity of my (car's) engine's running ' $E$ '). $R$ is a spatial part of E's running.

[12] The spatial region in which the rattling and knocking occurs is a spatial part of the spatial region in which E occurs. (true)

7. Matters are complicated by the fact that the terms for objects are often connected with several default organizations-for example, the ridge beam is a spatial part of a house, even though many houses do not have ridge beams.

8. While so-called 'granularity arguments' serve well to highlight transitivity failures of spatial parthood on arbitrary types of entities besides spatial regions, it is important to note that they involve a clandestine "aspect shift" from stuffs to mixtures and from activities to sequences of events, cf. Seibt (2004b, ch. 4). 
Sentences [8] and [11] will be judged false, I submit, once contrasted with [9] and [12], the only reading in which they could have appeared to be true- a certain amount of water W is a spatial part of the entity referred to by 'the gin-and-tonic in my glass' (whose functional structure F determines what counts as spatial part), but the amount of radon contaminating the water is at best a spatial part of the spatial region occupied by the mixture of chemical substances in my glass; similarly, the rattling $\mathrm{R}$ is at best a spatial part of the spatial region occupied by the complex of processes occurrent under the hood of my car, but not a spatial part of the running of my car's engine.

These transitivity failures of spatial parthood on objects, stuffs, and activities show that the strictly transitive part-relation of (CEM) cannot count as a formal reconstruction of spatial parthood per se, i.e., irrespective of the 'relata' of spatial parthood; at best, (CEM) formally captures spatial parthood defined on spatial regions. So we should reject (A4).

If (CEM) is not a formal reconstruction of spatial parthood per se, however, it also follows that assumption (A5) should be rejected: the part relation formally defined by (CEM) does not cover any part relation that is cognitively basic. But besides the illustrated transitivity failures of spatial parthood on objects, stuffs, and activities, there are also other reasons for giving up on the idea that a cognitively basic parthood relation is strictly transitive, as (CEM) requires. For perceptual immediacy in the sense of non-inferentiality holds not only for part-relations that we visually perceive, but also for part-whole relationships that we hear, smell, feel by touch, or are proprioceptively aware of. I hear the flute in the sound of the orchestra's playing; I smell 'apple' in the fragrance of your perfume; I feel the smoothness and the softness in the texture of my cat's fur; I feel how I move each leg in my walking. As mentioned above, phenomenologically speaking there seems to be a basic relationship of asymmetric 'belonging with' that holds across sensory modalities. But such basic, directly perceived 'belonging with' is subject to the context-sensitivity of perception and thus is not strictly transitive. When the flutist plays alone, the sound I experience has overtones, i.e., the overtones belong with the sounds she makes; but when the flutist plays the same melody within the orchestra, these overtones do not belong with the sound I experience from the orchestra. Similarly, as every cook knows, the taste of chili, which belongs with hot pepper eaten in isolation, disappears within curry with much cream and pineapple. I feel my weight shifting if I lift my left leg, but the weight shift does not belong with my propriosensory experience of walking. Some 
of these transitivity failures are due to contextivity or threshold effects in our sensory experience, some are due to physical or chemical interactions; for present purposes it only matters that the asymmetric 'belonging with' relationship that structures what we immediately experience in all sensory modalities is not strictly transitive.

In sum then, if one were to assume (A1) and link cognitive basicness to perceptual immediacy, we could indeed argue that there is a cognitively basic relationship of asymmetric 'belonging with,' but since this relationship is not transitive, we could not claim that (CEM) is a formal theory of such a generic basic part-whole relationship. At best, (CEM) is a formal theory of the application of a specific variety of this relationship: spatial parthood, to a specific domain: spatial regions, and the transitivity of spatial parthood on spatial regions results from the identity conditions of spatial regions, which are particularly weak.

As indicated by the subjunctive brackets of the considerations presented so far, I want to leave open here whether (A1) is a reasonable path to cognitive basicness, and, in fact, whether cognitive basicness is a useful criterion for the choice of the foundational relation in an ontological theory. My main reason for introducing this line of argument was to shake up the ubiquitous conviction among ontologists that the basic sense of 'part' is transitive and that (CEM) - while not sufficient to reconstruct all sorts of part-whole relationships-formally captures the basic sense of part (Varzi 2006:2). In his classic Parts Simons (1987) states that anyone who "seriously disagrees" with the formal properties of the part-relation: irreflexivity, asymmetry, and transitivity, "has failed to understand the word" (11) but at the same time stresses that "'part,' like other formal concepts, is not univocal, but has analogous meanings according to whether we talk of individuals, classes, or masses" (2). The general theoretical landscape he draws up is that there is a basic transitive part relation which in application to some domains ("classes and masses") fulfills an "extensionality axiom" (the Proper Parts Principle (PPP): that the item 'A' refers to has the same parts as the item referred to by ' $\mathrm{B}$ ' implies that ' $\mathrm{A}$ ' and ' $\mathrm{B}$ ' refer to the same entity, and vice versa); in application to other domains, such as continuants and integral wholes, this basic transitive part-relation is not "extensional" in the sense of the Proper Parts Principle. In this way any variations in the meaning of 'part' are associated with the identity axiom PPP and not with the transitivity axiom, which he takes to be indispensable.

There is an alternative, however. If the task of mereology is to articulate the logic of 'is part of' as reflected in common-sense and scientific 
reasoning, and if we find that the reasoning samples we work from imply that 'two' entities (e.g., a person and her body) have the 'same' parts or that 'one' entity (at different times) has different parts, we can attribute these phenomena either to the fact that parthood is not extensional in this domain or to the fact that it is not transitive. Consider the following example. One might argue that PPP does not hold since a person and her body are different entities but have the same parts. Presenting this example, which is due to F.C. Doepke, Simons explains:

The difference between a person and body is that the former only exists as long as certain kinds of process are going on in the body. These processes are not parts, nor are they relationships among the parts, though they are closely connected with such relationships (114).

As Simons' explanation brings out very clearly, counterexamples against PPP depend on which items we consider to be the parts of the items involved. However, to get the given counterexample to work the (i) clandestine restriction on the types of parts (e.g.: no processes) is actually not enough; one also needs to (ii) equate 'part' with 'spatial part' and (iii) equivocate 'person' with 'spatial region occupied by a person,' as well as 'human body' with 'spatial region occupied by a human body,' respectively. If the conceptual slide in (iii) were not present, the example would cease to be counterexample to PPP, since a person has as spatial parts all those parts of her body she can have concerns about (feel pain in or feel shame or pride about) but not the millions of cells that are part of her body.

In general, I think that all currently discussed putative counterexamples against the PPP make use of the transitivity of the part-relation and depend on two conceptual manipulations: (i) a reading of 'is part of' as 'is spatial part of' and (ii) an equivocation of the items involved with their spatial extensions. ${ }^{9}$ If we refrain from these conceptual manipulations and drop the idea that 'is part of' is a transitive relation, we can retain the PPP. ${ }^{10}$ Differently put, the inferential restrictions that we achieve by dropping PPP

9. Cf. Seibt $201+\mathrm{a}$.

10. Similarly for alleged counterexamples to PPP showing that it does not follow that A and $\mathrm{B}$ are different if $\mathrm{A}$ and $\mathrm{B}$ do not have all parts in common-e.g., the notorious hapless cat that loses its tail in an accident. To receive a counterexample here one needs to (i) again equate part with 'spatial part' and (ii) argue that A (the 'cat-at-t') has spatial-part tail, while B (the 'cat-at-t*') does not, and that $A$ and $B$ count as one and the same cat since they are parts of the life of that cat (according to the identity principle for continuants, a continuant is identical with all parts of its life-the cat is "wholly present" (D. Lewis) at any moment of its life). Again, if we reject that a spatial part of a part of the cat's life is a spatial part of the cat, the counterexample collapses. 
as a principle of identity can also be introduced if we drop the transitivity of parthood while retaining PPP. Before illustrating this latter option in the next section, let me conclude this section with a brief reflection on why most ontologists wish to hang on to the former option, i.e., endorse the transitivity of parthood and sacrifice the PPP.

As suggested above, if cognitive basicness is linked to phenomenal immediacy or non-inferentiality, there are part-whole relationships that have just as good a claim to cognitive basicness as spatial parthood on spatial regions, i.e., the relation that serves as prime illustration for the axioms of CEM. Spatial parthood on spatial regions is transitive, other phenomenally immediate part-relations - e.g., spatial parthood on things-are not transitive. Thus the focus on transitive parthood cannot be explained in terms of phenomenal immediacy alone. But why, then, has spatial parthood on spatial regions taken centerstage in mereology? Why is it, that even where considerations of phenomenal immediacy and cognitive basicness do not come into play at all, mereologists of all stripes-those who drop PPP, or those who acknowledge that in order to model material or functional parthood transitivity restrictions must be introduced-insists that the "basic" part-relation is transitive? ${ }^{11}$ Why is it that in the debate about transitivity of parthood, to the extent to which there has been any debate at all, proponents of transitivity charge opponents of transitivity with equivocating 'part' with 'functional part,' while the equivocations of the proponents of transitivity I have been highlighting here, of 'part' with 'spatial part', and of ' $N$ ' with 'spatial region of $N$,' have not yet been observed? ${ }^{12}$ Or again, why is it that the shortcomings of (CEM) for the modeling of the many different kinds of part-whole relationships-such as material parts, functional parts, construction parts, maintenance parts, design parts ${ }^{13}$ - have been almost exclusively addressed by relinquishing PPP, without explicit discussion that this strategy is just one of the options? Why has the theoretical juncture: 'extensionality' (or better: identity via parthood) versus transitivity' never come into sight? ${ }^{14}$

11. See e.g. Guizzardi (2009).

12. For the debate about transitivity see e.g. Cruse (1979), Winston (1987), Seibt (1990, ch. 5; 2004, ch. 4), Varzi (2006), Vieu (2006), Guizzardi (2009); for a further discussion of the equivocations involved in the transitivity debate see Seibt forthcoming.

13. On the varieties of parthood see Cruse (1979), Winston et al. (1987), Simons/Dement (1996), and Simons (2013).

14. I may have been the only one so far who has explored this route, since 1990 in a continuous series of papers devoted to exploring the mereology of processes (see e.g., 1995, 1996, 1999, 2000, 2001, 2004a, 2008, 2009, and in particular 2004b). 
A proper answer to these questions - which I can here only point to-would have two parts. The first part would reconstruct the historical origins of mereology during the first decades of the $20^{\text {th }}$ century, when mathematicians, metaphysicians, and ontologists (S. Lesniewski, A. N. Whitehead, N. Goodman, S. Leonard and others) searched for a formal theory that could replace set-theory in mathematics (geometry), logic, and the formal reconstructions of conceptual content in the sciences. Since the subset relation is transitive, a transitive parthood relation seemed most suitable; in addition, given the new program of rendering philosophical discourse more formal, basic domain relations needed some inferential strength. But the history of mereology can only explain the motivations for preferring a transitive relation. In order to explain why 'spatial part of $\mathrm{X}$ ' could be equivocated with 'spatial part of the spatial region occupied by $\mathrm{X}^{\prime}$ we would need to reconstruct, second, a longstanding research paradigm of the ontological tradition. This paradigm has its roots in the Aristotelian focus on substances, concrete particular individual things that persist in time, but received its decisive impulse in the Cartesian equation of material substances, res extensa, with geometric regions. If the spatial extension of an individual object is identified with a continuous geometric region, spatial parthood on objects clearly would appear to be transitive. But, as our examples above should have brought out, while an object extends over a spatial region, not every item in that region (i.e., not every item that is a spatial part of that region) is a spatial part of the object.

In short, in connection with the longstanding theoretical fixation of (Western) metaphysicians on enduring objects or substances, the Cartesian notion of geometric regions as the extensions of objects became an integral component of a comprehensive mindset that influenced the development of formal theories in philosophy, and to the present day dominates in the discussion of analytical ontology. The dogma of the transitivity of spatial parthood per se is just one reflection of the workings of the "myth of substance" - the presupposed ontological priority of objects-in combination with the Cartesian geometricalization of extensions, which also played into the hands of the idea that individual objects are 'particulars,' i.e., that they are necessarily uniquely located entities whose thisness (identity, individuality) is defined in terms of their location (at a time). ${ }^{15}$ If the identity

15. The "myth of substance" or the "substance paradigm" can be reconstructed as a network of about 20 constraints on category construction (see my 1990, 2005, 2008, and 2010). Lorenz Puntel identified the "object-ontological dogma" in analytical ontology already in the early 1980s; 
of an entity is given by its location, it can indeed seem straightforward to identify the extension of an entity with the geometric region occupied by that entity.

This move loses its plausibility once we turn away from substances and consider activities, such as the snowing in Aarhus on February $2^{\text {nd }}, 2014$ or the Royal Orchestra's playing on the Queens's $70^{\text {th }}$ birthday, i.e., entities that are spatially uniquely located but whose identity is not defined in terms of their location (for instance, because they are spatially superposable). These actitivies occupy (at each time $t$ in temporal interval $\mathrm{T}$ ) a geometric region $\mathrm{R}$, where $\mathrm{R}$ can be understood either as the collection of scattered regions that each are continuous and simply connected (the collection of regions occupied by each snow flake or each musician); alternatively, $\mathrm{R}$ can be understood as one multiply connected continuous region (the entire region with spatial holes where at $t$ no snow flake or musician is positioned). But no matter which of these two interpretations of $\mathrm{R}$ we adopt, since we determine the spatial parts of a snowing or of an orchestra's playing in relation to functional organization of these activities, clearly not every spatial part of the region $\mathrm{R}$ is a spatial part of these activities. The space occupied by a quarter of the nucleus of an $\mathrm{H}$-atom in the water molecule of a snowflake is not a spatial part of the snowing (though surely a spatial part of the spatial region occupied by the snowing).

In sum, then, if mereology is the philosophical analysis of part-whole relationships by means of a formal theory, then mereology has always been characterized by a basic theoretical juncture. The first option is to operate with a transitive part-relation and abandon the identity principle PPP to block unwanted identifications, rejecting what we have come to call the "extensionality" of classical mereology. The second option is to retain PPP but to block "extensionality" much earlier, by abandoning three ideas: (i) the idea that the extension of an item is the geometric region occupied by the item, (ii) the idea that the only basic sense of parthood is 'spatial part' since it is cognitively basic in some sense, and (iii) the idea that spatial parthood per se, on arbitrary domains, is a transitive relation. The myth of substance has obscured the second option; as I will now argue, it is an option worth exploring.

for the first systematically worked out proposal of a metaphysics free from the relevant dogmatic restrictions see Puntel (2010). 


\section{Leveled mereology}

As suggested above, there is a phenomenally immediate and, in this sense at least, cognitively basic relationship of asymmetric 'belonging with' that structures what we perceptually take in. Such asymmetric 'belonging with' is what we express, I want to suggest, when we use 'part of' in everyday speech, as a relationship that can be applied to any entity type: things, institutions, activities, events, actions, stuffs, collections, features. ${ }^{16}$ 'Belonging with' is the most generic and poorest sense of 'part of,' an asymmetric association of items in the widest sense that can be further specified in spatial, temporal, material, functional, operational regards. Formally this basic and generic sense of 'is part of' as 'belongs with' must be formally modeled by a non-transitive relation-there are some transitivities along the chains of 'belongs with' but the depth of parthood chains where the inferential pattern of transitivity can be applied depends on the specification of the part-relation involved (spatial, temporal, material, functional etc.) and on the kind of relata.

In tandem with the theoretical juncture mentioned above, i.e., the choice between 'retain transitivity and drop PPP' versus 'retain PPP and drop transitivity,' there is a choice between two strategies for the modeling of transitivity restrictions for specifications of the part-relation, e.g., 'functional part' or 'construction part.' We can-and this is the common strategy among mereologists currently-begin with a transitive part-relation and restrict its transitivity for certain specifications and domains. ${ }^{17}$ Alternatively, we can operate with a non-transitive part-relation and introduce transitivity for certain specifications and domains (in fact, in my view, only for the very limited case of spatial parthood on spatial regions).

The formal framework of 'belongs with', or of 'part of' in its most basic and generic sense, operates best, in my view, with the second strategy. Let us look at some basic axioms and definitions of such a frame-

16. Compare the following examples, taken from the web: "Blogging is part of life"; "Russia is part of the West"; "Music is part of God's Universe"; "All I see is part of me" (book title); "Learning to negotiate is part of the advocacy process"; "My heritage is part of who I am"; "Is part of your college education missing?"; "Having a good signed contract is part of running a professional business"; "Boston is part of the Islam"; "Syria is part of the solution"; "Looking immaculate is part of what I do": "Pain is part of running a marathon"; "Hopping too is part of running"; "Fab Face is part of Screaming Talent"; "The concert is part of the 11th Ludwig v. Beethoven Easter festival."

17. Cf. e.g., Simons (1987; 2013); Varzi (2006). 
work, which I call 'Leveled Mereology' (LEM). ${ }^{18}$ The part-relation of LEM, symbolized by ' $G$,' is non-transitive (Ax1), asymmetric (Ax2), and irreflexive $(\mathrm{Ax} 3)$ :

$$
\begin{aligned}
& (\mathrm{Ax} 1) \neg[(\mathrm{x} \subset \mathrm{y} \& \mathrm{y} \subset \mathrm{z}) \rightarrow \mathrm{x} \subset \mathrm{z}] \\
& (\operatorname{Ax} 2)(\mathrm{x} \subset \mathrm{y}) \rightarrow \neg(\mathrm{y} \subset \mathrm{x}) \\
& (\operatorname{Ax} 3) \neg(\mathrm{x} \subset \mathrm{x})
\end{aligned}
$$

Since ' $G$ ' is non-transitive according to $(A x 1)$, the relationships established by $(A \times 2)$ and $(A \times 3)$ reach only the 'immediate parts' of an entity, that is, the parts at the first level of the partition of an entity. For example, a whole $\alpha$ cannot be part of itself, in accordance with (A3), but it may well be part of ... part of itself. Similarly, if $\alpha$ differs from $\beta$ and has $\beta$ as part, $\alpha$ cannot be part of $\beta$, in accordance with (A2), but $\alpha$ may well be part of ... part of $\beta$. As I shall briefly elaborate below, the particular interest of LEM lies precisely in the fact that it allows for such 'parthood loops,' but let us first consider some definitions that will allow us to extend the logical properties of ' $C$ ' in a differentiated fashion. Every basic term (individual constant) of (LEM) is associated with a default partition $\mathrm{P}(\mathrm{x})$; e.g., let the term ' $\alpha$ ' be associated with the partition $\mathrm{P}(\alpha)$, which has three levels ${ }^{19}$ :

18. I have envisioned and sketched Leveled Mereology for some time, always in application to processes (cf. Seibt 1990 ch. 5; 2001, 2004 [with unfortunate misprints in the formulas]; more worked out versions are in Seibt 2004b, ch. 4; Seibt 2008, and, in particular, in Seibt 2009).

19. For example, $P(\alpha)$ might represent the relationships in generic action contexts such as: removing the flat is part of changing a tire, loosening the lug nuts is part of removing the flat tire, turning the wrench is part of loosening the lug nuts. Alternatively, the partition can be taken to represent a scientific partition, e.g., an anatomical relationship: actin myofilament is part of a myofibril, which is part of a muscle fiber, which is part of a muscle. Note that the aim of LEM is to reconstruct the mereological inferential meaning of common sense and scientific kind terms; thus the structure of the partitions are given by relevant pieces of (common sense or scientific) discourse involving the kind terms in question. 


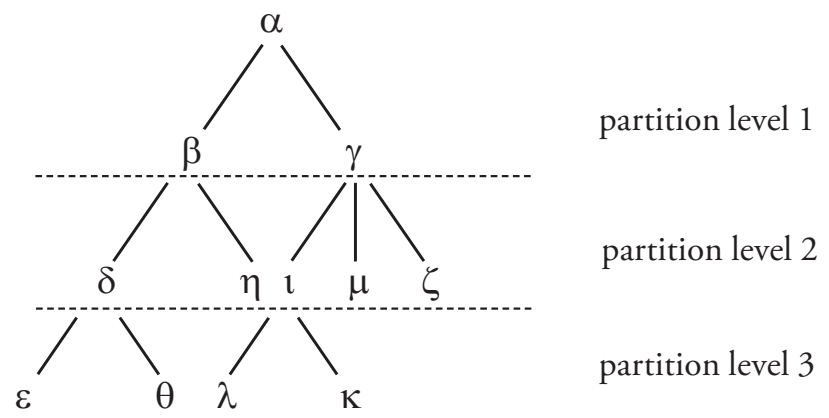

Figure 1: Partition with three levels of nodes

To introduce the notion of a partition and a partition level, we first define parthood resulting from the $n$-th iteration of ' $\hookrightarrow$ ':

(D1a) $x G_{n} y \leftrightarrow$ for all $n$ in an ordered sequence of natural numbers of length $\mathrm{N}$ :

if $n=1$ then $\mathrm{x} \subset_{\mathrm{n}} \mathrm{y} \leftrightarrow \mathrm{x} \subset \mathrm{y}$

if $n>1$ then $\mathrm{x} \subset_{\mathrm{n}} \mathrm{y} \leftrightarrow \exists \mathrm{z}\left(\mathrm{x} \subset \mathrm{z} \& \mathrm{z} \subset_{\mathrm{n}-1} \mathrm{y}\right)$

The relation ' $\mathrm{x} \mathcal{G}_{\mathrm{n}} \mathrm{y}$ ' (read: ' $\mathrm{x}$ is $\mathrm{n}$-part of $\mathrm{y}$ ') indicates the level of mereological embedding of one item in another, but is always relative to a given partition..$^{20}$ For example, in figure 2 process $\lambda$ is 3-part of $\alpha$ and 2-part of $\gamma$. The $n$-parts of an entity $\alpha$ are those entities y for which ' $y \subset_{n} \alpha$ ' holds, relative to a given partition:

(D1b) $\mathrm{x}$ is $n$-part of $\mathrm{y}=_{\mathrm{df}} \mathrm{x} \subset_{\mathrm{n}} \mathrm{y}$

Note that n-part is not a transitive relation. In order to define a partrelation that is transitive across partition levels down to the $\mathrm{N}$-th level of the partition, we need to add a transitivity postulate (here omitted) for the relation ' $G_{\text {trans-N }}$ '.

(D1c) $\mathrm{x} \subset_{\text {trans-N } \mathrm{N}} \mathrm{y} \leftrightarrow \mathrm{x} \subset_{\text {y or } \mathrm{x}} \subset_{n} \mathrm{y}$, for any $1<\mathrm{n} \leq \mathrm{N}$

20. Not all partitions are finite; the identities of nodes of infinite partitions can be preserved by means of LEM's relativized identity axioms, see below (Ax4a). 
For a given entity $\alpha$, any entity y in the domain of the relation ' $y G_{i \leq n} \alpha$ ' belongs to the so-called $n$-partition of $\alpha$. In contrast, any entity y in the domain of ' $y \hookrightarrow_{n} \alpha$ ' (the $n$-th iteration of ' $\subseteq$ ') belongs to the so-called partition-at-level- $n$ of $\alpha$. That is, the $n$-partition of $\alpha$ comprises entities at all partition levels up to and including level $n$, while the partition-at-level-n of $\alpha$ denotes entities at one level only. Items at level $\mathrm{n}$ of a partition $\mathrm{P}(\mathrm{x})$ are called the n-parts of $\mathrm{x}$ (see D1b), items below or above a certain 'threshold' can be easily referred to by quantifying over the ' $>n$-parts' or ' $<k$-parts' of $\mathrm{x}$ in $\mathrm{P}(\mathrm{x})$, respectively. For example, in figure 1 above the '2-parts' of $\alpha$ are $\beta$ and $\gamma$, and the '>2-parts' of $\alpha$ are $\varepsilon, \theta, \lambda, \kappa$.

There are two sorts of mereological relationships that can be stated with the framework of (LEM): (i) universal relationships holding for any partition, either at levels, or across levels, or for certain thresholds (e.g., relative to certain domains of application); (ii) partition-relative relationships, holding only within a given partition or collection of partitions. Statements about partition-relative relationships may be made in two ways, however. They may relate to a certain partition level $n$ of $\mathrm{P}(\mathrm{x})$ where $\mathrm{x}$ is the uppermost node; in some contexts it is important, however, to be able to address relationships relative to sub-partitions $\mathrm{P}(\mathrm{y})$ in $\mathrm{P}(\mathrm{x})$, where $y G_{n} x$ in $P(x)$. The expression ' $P\left(y G_{n} x\right)$ ' denotes a subpartition $\mathrm{P}$ of the partition of $\mathrm{x}$ that has $\mathrm{y}$ as its head $\operatorname{node}^{21}$; if $\mathrm{z}$ is a $\mathrm{m}$-part of $P\left(y G_{n} x\right)$, $z$ is also a $m+n$-part of $x$. The simple index ' $n$ ' on the part-relation ' $G_{\mathrm{n}}$ ' indicates that a LEM-formula addresses an absolute partition level of a partition $\mathrm{P}(\mathrm{x})$, while a composite index ' $\mathrm{C}_{\mathrm{m}+\mathrm{n}}$ ' indicates that a LEMformula addresses a partition level of any sub-partition $P\left(y G_{n} x\right)$. Finally, the composite index ' $G_{\mathrm{m}(\mathrm{y})+n}$ ' is used to identify m-parts within the subpartition $\mathrm{P}\left(\mathrm{y} \subset_{\mathrm{n}} \mathrm{x}\right)$ for some specific $\mathrm{y}$ that is $\mathrm{n}$-part of $\mathrm{P}(\mathrm{x})$. For example, in figure 1 above, the following relationships hold: $\theta \complement_{3} \alpha ; \theta, \lambda, \subset_{2+1} \alpha$; and $\theta \subset_{2(\beta)+1} \alpha .^{22}$

The distinction between relative and absolute partition levels in a partition also matters for a proper understanding of the anti-symmetry of the part-relation ' $\subseteq$ '. Axioms ( $\mathrm{Ax} 2)$ and $(\mathrm{Ax} 3)$ hold at any (absolute) partition level $\mathrm{n}$ in a partition $\mathrm{P}(\mathrm{z})$, i.e., for any given $n$ in a partition $\mathrm{P}(\mathrm{z})$ with $\mathrm{n}$-parts $\mathrm{x}$ and $\mathrm{y}, \mathrm{x} \subset_{\mathrm{n}} \mathrm{y} \rightarrow \neg\left(\mathrm{y} \subset_{\mathrm{n}} \mathrm{x}\right)$, but this does not hold across variable $n$. This is ensured by $(A x 4)$ :

21. For the sake of simplicity I assume here that y occurs only once in $\mathrm{P}(\mathrm{x})$.

22. One of the main tasks of (LEM) in application to processes is to investigate how absolute partition levels map into relative partition levels-i.e., how much of the mereological structure of a process is preserved once it becomes part of another. 
$(\mathrm{Ax} 4) \mathrm{x} \subset_{\mathrm{n}} \mathrm{z} \& \mathrm{y} \subset_{\mathrm{n}} \mathrm{z} \rightarrow\left[\neg\left(\mathrm{x} \subset_{1(\mathrm{y})+\mathrm{n}} \mathrm{z}\right) \& \neg\left(\mathrm{y} \subset_{1(\mathrm{x})+\mathrm{n}} \mathrm{z}\right)\right]$

If $\mathrm{x}$ and $\mathrm{y}$ are $\mathrm{n}$-parts of the same partition $\mathrm{P}(\mathrm{z}), \mathrm{x}$ cannot be part of $\mathrm{y}$ in this partition, and vice versa. But note that $(A x 4)$ leaves room for the possibility that $\mathrm{x}$ and $\mathrm{y}$ are parts of parts of ... parts of each other, i.e., mutually contain each other at deeper levels of respective sub-partitions of a main partition. For example, relative to a sub-partition $P\left(\alpha C_{i} \gamma\right)$ it may hold that $\beta \subset_{3} \alpha$, and relative to a sub-partition $P\left(\beta \subset_{k} \gamma\right)$ it may hold that $\alpha C_{3} \beta$. In a sense, then, for such a partition $\mathrm{P}(\gamma)$ one may claim that $\alpha C_{3} \beta$ and $\beta C_{3} \alpha$, but only in the sense that ' $C_{3}$ ' in each case is relative to two different sub-partitions of $\mathrm{P}(\gamma)$, not as a statement about the absolute partition levels of $\mathrm{P}(\gamma) .^{23}$

Perhaps the most important feature of (LEM) is that PPP, the identity principle, is defined across partitions but relative to a partition level, with the following two axiom schemas, as identity at a level $n$ (see Ax5a) and identity relative to partition levels up to depth $n$, (see Ax5b, where ' $G_{\leq n}$ ' is used to abbreviate the restricted quantification over partition levels, read 'for each $m \leq n$ '):

(Ax5a) $\forall \mathrm{z}\left(\mathrm{z} G_{\mathrm{n}} \mathrm{x} \leftrightarrow \mathrm{z} \subset_{\mathrm{n}} \mathrm{y}\right) \leftrightarrow \mathrm{x}==_{\mathrm{n}} \mathrm{y}$

$($ Ax $5 b) \forall z\left(z G_{\leq n} x \leftrightarrow z C_{s n} y\right) \leftrightarrow x==_{s n} y$.

Two terms are coreferential (in common ontological parlance: 'two entities are identical') if and only if they have the same $n$-parts or $\leq n$-parts. These two identity principles account for the context-relativity of our judgments of sameness; in some contexts coarse-grained comparisons suffice, while others require an in-depth investigation. For example, in certain contexts it seems correct to identify the rotating of a metal disk with its heating up, or a performance of certain bodily movements with a greeting, or a singing with a production of noise, or a house with shelter, and such coarse-grained (subsumptive) identifications take their bearings from a cursory survey of what is involved or part of being a certain rotating of a disk, greeting, singing, or house. In other contexts, however, we

23. Again, that it might be desirable to have a formal framework where one can express, very generically, that two items are part of one another (e.g., trigger or modify one another) become most plausible if one applies (LEM) to processes (cf. my 2001, 2004a, b, 2009). Cotnoir (2010) provides additional motivations for accepting parthood-loops, but chooses to give up anti-symmetry altogether, while retaining transitivity. 
will look more closely into deeper levels of the partitions of these items and find mereological incongruences in terms of which the items can be kept apart. ${ }^{24}$

Finally, to complete our sketch of the basic axioms of (LEM), given that ' $C$ ' is a non-transitive relation we can diversify the part-relation modeled by operating with ' $G$ ' instead of ' $G$ ' in various axioms and definitions. ${ }^{25}$ For example, we can distinguish various degrees of overlap as well as three forms of 'non-overlap' among processes: discreteness reaches only into the first partition level, disjointness expresses non-overlap across levels, and divergence establishes non-overlap at a certain partition level. The supplementation principle in (LEM) is defined as a 'shallow' constraint in terms of discreteness (symbol: ' $\int$ ') rather than disjointness or divergence:

$$
\begin{aligned}
& (\mathrm{A} 6 \mathrm{a}) \mathrm{x} \subset \mathrm{y} \rightarrow \exists \mathrm{z}\left(\mathrm{z} \int \mathrm{x} \& \mathrm{z} \subset \mathrm{y}\right) \\
& (\mathrm{A} 6 \mathrm{~b}) \mathrm{x} \subset_{\mathrm{n}} \mathrm{y} \rightarrow \exists \mathrm{z}\left(\mathrm{z} \int_{\mathrm{n}} \mathrm{x} \& \mathrm{z} \subset_{\mathrm{n}} \mathrm{y}\right)^{26}
\end{aligned}
$$

With such a weak version of the supplementation principle wholes may have 'non-overlapping yet entangled' parts, i.e., parts at partition level $n$ that do not overlap at level $\mathrm{n}+1$ but share parts at some partition levels below the second level.

\section{Emergent parts of processes}

The preceding brief sketch of (LEM) is mainly intended as an illustration of Leveled Mereology as a general formal strategy; the idea of a partitionbased mereology, the new notions of parthood-at-level-n and parthoodup-to-level-n, and the relativization of the identity principle have a host of fruitful applications for the traditional problems of ontology (material constitution, persistence and change, individuation of events and actions). Moreover, there are many aspects of Leveled Mereology that provide useful connections between, on the one hand, the investigation of formal mereol-

24. This cognitive capacity of 'zooming in and out,' or moving down and up a partition to reason about more or less specific entities, we systematically exploit in many pragmatic contexts, from planning to excuses.

25. I am omitting here a discussion of certain constraints to be observed by such diversifications of axioms, for details see Seibt $(201+b)$.

26. Discreteness at level $n$, i.e., ' $\int_{n}$ ' is defined as follows: $x \int_{n} z={ }_{d f} x \subset_{n} y$ and $z \subset_{n} y$ in $P(y)$ and there is no w for which ${ }_{w} \subset_{1(x)+n} y$ and ${ }_{w} \subset_{1(z)+n} y$ (i.e., w is 1-part in the sub-partitions $\mathrm{P}\left(\mathrm{x} \subset_{\mathrm{n}} \mathrm{y}\right)$ just in case it is not 1-part in the sub-partition of $\mathrm{P}\left(\mathrm{z} \subset_{\mathrm{n}} \mathrm{y}\right)$, and vice versa). 
ogy in ontology, and, on the other hand, the study of partitioning frames used in scientific explanations. ${ }^{27}$

I have explored the strategy of Leveled Mereology mainly in application to processes. In a recent publication Peter Simons writes: "Relatively little thought has however gone into the question whether we can simply adapt the mereology coming from mathematics and logic for processes" (2013: 161). I choose to read this statement as a claim about numbers of analytical ontologists engaged in such a project, and in this sense I can only agree. ${ }^{28}$ The mereology of processes is a particularly difficult topic, in my view, for four reasons. First, we reason about processes (activities, events, developments, actions) in ways that defy ontological categorization in terms of the traditional category dualism of 'concrete, determinate, particular, and individual' entity versus 'abstract, indeterminate, general' entity - processes are best conceived as concrete, non-particular, indeterminate individuals. This new category I call dynamics. ${ }^{29}$ Second, processes stand in non-transitive part-relations. Third, complex processes exhibit both sequential, circular, and multiply entangled structures (feedback, multiple entangled feedback); in order to accommodate non-sequential

27. Cf. Winther (2011, fn. 3), where this desideratum is mentioned. Winther's account of scientific part-whole explanations, based on the claims that "there are multiple cross-cutting manners of abstracting a system into kinds of parts-i.e., there are multiple partitioning frames" (2011:397) and that "parts are abstracted through partitioning frames closely linked to explanatory projects" (400) fit directly with the constructive strategies of LEM, namely, (i) to begin with the most generic part-relation terms of which specific part-relations (functional, structural, or even more specific: morphological, physiological etc.) can be defined; (ii) to operate with a partrelation that-unlike transitive 'part-of'-does not imply any implicit domain restrictions and allows, for instance, genes in the role of "structure-parts" and "activity-parts" (cf. ibid. 412); (iii) to associate terms with default partitions. I will need to leave the elaborations of these connections for another occasion, but note that Leveled Mereology also contains rules for operations on partitions (extension, reduction, rebranching, insertion, deletion) to model changes in the conceptual resources on which mereological reasoning is based, from simple predication to change of context to scientific progress.

28. Mereological relationships for processes also have been investigated in philosophy of science, especially in philosophy of chemistry (cf. e.g. Needham 2003) and biology (cf. e.g. Kaiser 2014; Winther 2012), but, as far as I can see, in analytical ontology the mereology of processes is largely uncharted; Mark Steen has explored logical relationships between stuffs and processes, Joseph Brenner developed a dialectical logic of processes (2008). I have addressed the issue from 1990 onwards, while working out the conceptual foundations for a new ontological category of non-particular concrete individuals called 'dynamics' or 'general processes' (cf. in particular 2004b ch. 4 and 5, but also 1990 ch. 5, 1995, 1997, 1999, 2000, 2001, 2004a, 2008, 2009, 2010).

29. In earlier terminological guises: 'dynamic masses,' 'free processes,' or 'general processes'; for book-length arguments for this categorization (cf. in particular my 1990 and 2004b). 
process architectures, a mereology of processes must allow for partition loops. Fourth, complex processes generate emergent processes that we may have reason to identify as 'new' parts of the complex process, or of one of its parts. It is this fourth 'challenge' for a mereology that I want to address in the following. ${ }^{30}$

In the longstanding debate about emergence, the keywords 'self-organization,' 'self-maintenance,' and 'complexity' have come to characterize systems exhibiting a behavior that can count as emergent in a fairly strong sense- the specifics of these behaviors are (physically) unpredictable even if they result from deterministic processes. Typical examples for such emergent behaviors are the development of convection cells (hurricanes, Benard cells), the joint movement of swarms, recurrent patterns in cellular automata, the differentiation of biological cell, a burning candle. Systems in which 'self-organizing,' 'self-maintaining,' or 'complex' behaviors occur have many different architectures; for present purposes I want to distinguish two sorts of emergence, simple and generative emergence. In cases of the first type, simple emergence, the emergent process does not itself causally influence the conditions for its continued existence-it is a product of an interaction of parts of the process system $S$ that affects parts of $S$ that are not identical with $S$. This type of emergence can be illustrated by C. Huygens' virtual metronome. As Huygens noted, if several pendulum clocks are hung on one suspended beam, after a time the pendulums become synchronized and swing more evenly. ${ }^{31}$ Let us describe the virtual metronome as a system of processes, using the folk-physical terms of common-sense reasoning. A relevant portion of the partition for such a system $(\theta)$ could look as follows, reducing the numbers of clocks to three:

30. As I hinted at above, partitions structured by the part-relation of LEM can contain loops and 'mutual containments,' provided these are not direct but mediated by a chain of part-of relations.

31. William Wimsatt cites Huygens' "virtual metronome" as an illustration for what he calls "weak emergence": "An emergent property is—roughly—a system's property which is dependent on the mode of organization of the system, without being embedded in any part of the system per se" (Wimsatt 1994, 373). Wimsatt's assessment that there is "nothing antireductionist" (ibid.) about the virtual metronome and similar cases of weak emergence depends on his assumption that the mode of organization is not part of the system. 


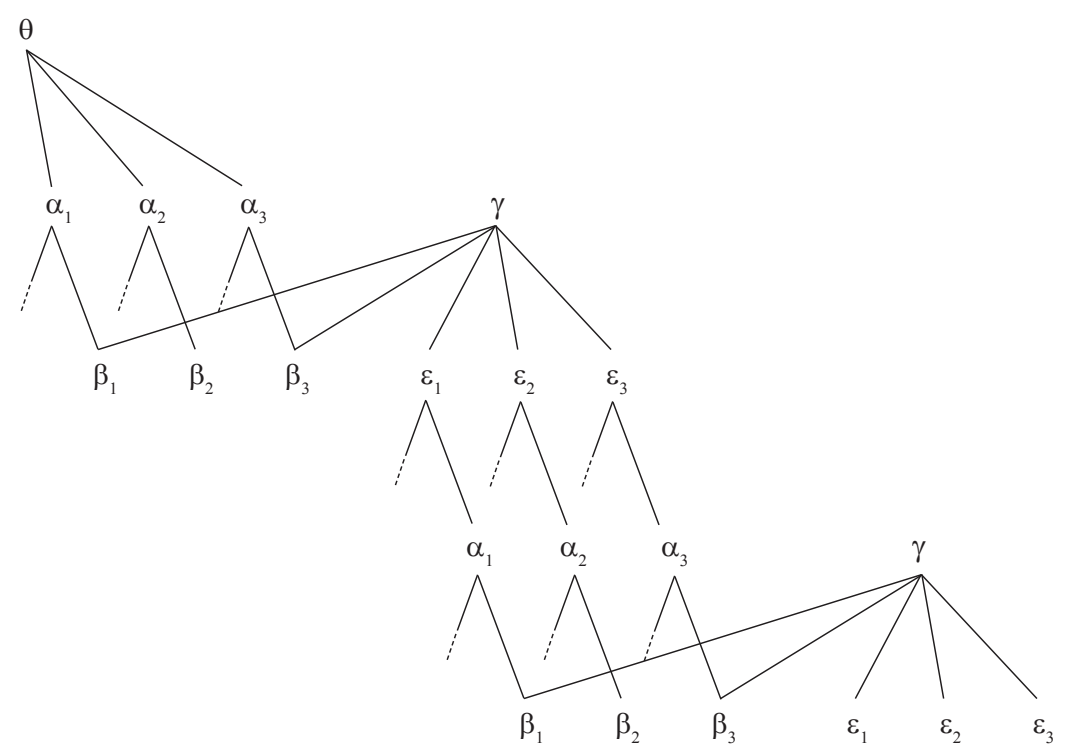

Figure 2: A (very simplified) analysis of virtual metronome $\theta$, in folk-physical' terms. $\alpha_{i}=$ swinging of a p endulum in location $i ; \beta_{i}=$ horizontal shifting of the beam in location $i$ (i.e., transmitting horizontal momentum); $\gamma=$ the interaction of the horizontal shiftings of the beam; $\varepsilon_{i}=$ dampening or accelerating of the pendulums (transmitting the momentum of the overall movement of the beam onto the oscillations) in location $i$.

Using ' $G_{n}$ ' we can introduce in LEM the definitions of sum-at- $n$ and sum-up-to-n relative to the $\mathrm{n}$-th partition level of $\mathrm{P}(\mathrm{x})$, which allows for the possibility of introducing terms for items that consist of the sum-at-n (or the sum-up-to-n) in $\mathrm{P}(\mathrm{x})$ and have additional parts that are not $\mathrm{n}$-parts (or $\leq \mathrm{n}$-parts) of $\mathrm{x}$. Let us call such items 'interactions,' and the relevant additional parts they introduce 'interaction product terms.' For example, in Figure 2, $\gamma$ is an interaction, consisting of a sum-at- 2 in $P(\theta)$, i.e., a sum of 2-parts of $\mathrm{P}(\theta)$, and the interaction product term $\delta$; there is a second occurrence of $\gamma$ in $\mathrm{P}(\theta)$, namely as sum of 5-parts of $\mathrm{P}(\theta)$. The iteration of a part of process system is indicative of simple emergence with feedback.

The second type of emergence, generative emergence, is typical of self-maintaining systems - the emergent complex process, e.g., a burning candle, causally contributes to the continued occurrence of its component processes (far-from-equilibrium), e.g., the heating of the wax or the perco- 
lating of the wax within the wick. ${ }^{32}$ Represented with the tools of LEM, the partitions of systems with generative emergence display a distinctive iteration structure involving the headnode $\mathrm{x}$ of the partition $\mathrm{P}(\mathrm{x})$ within subpartitions of $\mathrm{P}(\mathrm{x})$. To use again the case of self-maintenance, a process system $S$ includes a self-maintaining process, just in case:

(i) the partition $\mathrm{P}(\mathrm{S})$ contains at least one interaction $y$, with y being the sum of $z_{1} \ldots z_{k}$ and $S$, where each of the $z_{1} \ldots z_{k}$ is $2 \leq n$-part of $\mathrm{P}(\mathrm{S})$ for some given $\mathrm{n}$; and $\mathrm{S}$ is an interaction product term of $\mathrm{y}$;

(ii) $y G_{m(S)+n} S$, i.e., the interaction y is an $m$-part $(2 \leq \mathrm{m})$ of a subpartition with head node $S$ that is embedded in $\mathrm{P}(\mathrm{S})$.

Roughly speaking, the hallmark of partitions for process systems with productive emergence is that they have embedded partitions that are 'selfsimilar' in the sense that they contain structural repetitions involving the head-node of the embedded partition. It should be noted, though, that due to the fact that in LEM the identity of terms is defined relative to (i) the levels of (ii) a (relative/embedded or absolute) partition (see Axiom schema 5), we can choose whether system with generative emergence should be described as containing structural repetitions (choosing weak identity conditions for the terms of partition) or containing structural similarities (choosing strong identity conditions), so as to invite or avoid, respectively, idioms of 'self'-maintenance. ${ }^{33}$

\section{Conclusion}

I have argued in this paper for the use of a non-transitive generic partrelation that fits the most basic and indiscriminative usage of 'part-of' in the sense of 'belonging with' in common-sense reasoning. I suggested that the apparent naturalness of transitive reconstructions of 'part-of' is due to a combination of two factors. On the one hand, it reflects the historical provenance of formal mereology in the debate about the foundations of mathematics at the beginning of the $20^{\text {th }}$ century; on the other hand, it

32. Cf. Bickhard (2003). The example of the burning candle is Bickhard's illustration; it is discussed in some more detail in Seibt (2009).

33. Different degrees of self-similarity and types of emergence require various different types of adjustment on the 'reach' of ' $\subseteq$ ' in the axioms; for example, in partitions representing strong emergence (A2) ad (A5) need to have the same reach. For details cf. Seibt $(201+b)$. 
corresponds with a longstanding theoretical habituation to think of individuals as particulars, i.e., entities that can be individuated in terms of their spatial location (at a time) alone. This mindset, which I call the 'substance paradigm' or the 'myth of substance,' facilitates the equivocation of a thing with the region that it occupies. Only if we allow for this equivocation, I argued, it will appear that spatial parthood, which is transitive on spatial regions, is also per se transitive.

Once we operate with a non-transitive part-relation, new theoretical strategies for mereology are opening up. I sketched one of these strategies, called Leveled Mereology, which I illustrated with a particular implementation LEM. LEM can be used for all domains but also, in particular, for generic (non-particular) individuals such as processes. I pointed at some of the potential merits of LEM for the modelling of parthood relationships on process architectures, in particular the possibility to work with partitions that have 'loops,' representing feedback or the entanglement of processes. In LEM one can quantify in a differentiated fashion over parts at a partition level, which makes it straightforward to represent interactions within in a partition; I explored this feature briefly for the representation of parts of processes that are emergent in two senses of this term.

The turn to Leveled Mereology signifies a rather momentuous reorientation, however, with respect to the aims of mereology. Classical mereology, in particular (CEM), corresponds to a view of mereology as a logic or formal theory of reasoning, where a few axioms and definitions suffice to construct inferences based on the main connective (part-of) alone, deriving a large number of theorems. This conception is somewhat compromised already by intensional mereologies, where 'material' aspects enter by way of identity principles for certain domains and additional axiom sets for specifications of the main connective (functional part, construction part etc.). The strategy of Leveled Mereology in effect takes mereological reasoning to be about pairs $<\mathrm{x}, \mathrm{P}(\mathrm{x})>$ of terms and partitions, and thus leads even farther away from logic and into 'knowledge representation.' As long as the partitions of LEM operate merely with the generic relation of 'part-of,' few theorems can be derived; once more specific types of parthood (material, functional, design, construction, repair parts etc.) are defined in terms of this generic relation and for a certain region of the ontological domain, a host of specific inference principles can be stated. In short, the interest of LEM lies in the clarification and discussion of claims about mereological structures (especially for processes). LEM contains a few principles that 
characterize the generic notion of 'part' but is designed as a general formal framework for the representation of regional (context-sensitive) mereological knowledge and regional principles of mereological inference.

\section{REFERENCES}

Bickhard, Mark H. 2003: "Process and Emergence: Normative Function and Representation". In: Johanna Seibt (ed.), Process Theories. Netherlands: Springer, 121-155.

Brenner, Joseph E. 2008: Logic in Reality. Netherlands: Springer.

Cotnoir, Aaron J 2010: "Anti-Symmetry and Non-Extensional Mereology". The Philosophical Quarterly 60 (239), 396-405.

Cruse, D. Alan 1979: "On the Transitivity of the Part-Whole Relation". Journal for Linguistics 15(1), 29-38.

Dowty, David 1977: "Toward a Semantic Analysis of Verb Aspect and the English 'Imperfective' Progressive”. Linguistics and Philosophy 1(1), 45-78.

Doepke, Frederick 1982: "Spatially Coinciding Objects". Ratio 10(24), 45-60.

Guizzardi, Giancarlo 2009: "The Problem of Transitivity of Part-Whole Relations in Conceptual Modeling Revisited". In: Advanced Information Systems Engineering. Berlin Heidelberg: Springer, 94-109.

Mourelatos, Alexander 1978: "Events, Processes, and States". Linguistics and Philosophy 2(3), 415-434.

Needham, Paul 2004: "Continuants and Processes in Macroscopic Chemistry". Axiomathes 14(1), 237-265.

Puntel, Lorenz B. 2010: Structure and Being: A Theoretical Framework for a Systematic Philosophy. University Park, PA: Penn State Press.

Sanford, David H. 1993: "The Problem of the Many, Many Composition Questions, and Naive Mereology". Noûs 27(2), 219-228.

Seibt, Johanna 1995: „Individuen als Prozesse: Zur prozess-ontologischen Revision des Substanzparadigmas". Logos 2, 352-384.

- 1990: Towards Process-Ontology: A Critical Study of Substance-Ontological Premises. Ph.D. Dissertation at the University of Pittsburgh. Michigan: UMI-Press. To appear in a revised version as: The Myth of Substance. Berlin: DeGruyter.

- 1996: "Existence in Time: From Substance to Process". In: Jan Faye, Uwe Scheffler \& Max Urs (eds.), Perspectives on Time. Boston Studies in Philosophy of Science. Dordrecht: Kluwer, 143-182.

— 1999: "Dinge als Prozesse". In: Rafael Hüntelmann \& Erwin Tegtmeier (eds.), Neue Ontologie und Neue Metaphysik. Köln: Academia Verlag, 11-41. 
Seibt, Johanna 2000: "The Dynamic Constitution of Things". In: Jan Faye, Uwe Scheffler \& Max Urchs (eds.), Things, Facts and Events. Poznan Studies in Philosophy of Science. Amsterdam: Rodopi, 241-279.

- 2001: "Formal Process Ontology". In: Chris Welty \& Barry Smith (eds.), Formal Ontology in Information Systems: Collected Papers from the Second International Conference. Ogunquit: ACM Press, 333-345.

— (Ed.) 2003: Process Theories_Crossdisciplinary Studies in Dynamic Categories. Dordrecht: Kluwer.

- 2004: "Free Process Theory: Towards a Typology of Processes". Axiomathes $14(1), 23-57$.

- 2005a: General Processes—A Study in Ontological Category Construction. Habilitationsschrift at the University of Konstanz, Germany.

— 2005b: "Der Mythos der Substanz". In: Käthe Trettin (ed.), Substanz-Neue Überlegungen zu einer klassischen Kategorie des Seienden. Frankfurt: Klostermann, 197-229.

— 2008: "Beyond Endurance and Perdurance: Recurrent Dynamics". In Christian Kanzian (ed.), Persistence. Frankfurt: Ontos Verlag, 121-153.

- 2009: "Forms of Emergent Interaction in General Process Theory". Synthese 166 (3), 479-512.

- 2012: "Process Philosophy". The Stanford Encyclopedia of Philosophy. Edward N. Zalta (ed.), URL = <http://plato.stanford.edu/archives/fall2013/entries/ process-philosophy/>.

- 2014: "Transitivity". In: Hans Burkhardt, Johanna Seibt \& Guido Imaguire (eds.), Handbook of Mereology (forthcoming). München: Philosophia Verlag.

— 201+a: "Are There Transitive Part-Relations?" (under submission).

— 201+b: "Leveled Mereology" (under submission).

Simons, Peter \& Dement, Charles 1996: "Aspects of the Mereology of Artifacts". In: Roberto Poli \& Peter Simons (eds.), Formal Ontology. Dordrecht: Kluwer, 255-276.

Simons, Peter 1987: Parts: A Study in Ontology. Oxford: Oxford University Press.

- 2000: "How to Exist at a Time When You Have No Temporal Parts". The Monist (83)3, 419-436.

— 2013: "Varieties of Parthood: Ontology Learns from Engineering". In: Philosophy and Engineering: Reflections on Practice, Principles and Process. Netherlands: Springer, 151-163.

Varzi, Achille 2006: "A Note on the Transitivity of Parthood". Applied Ontology 1 (2), 141-146.

Vieu, Laure 2006: "On the Transitivity of Functional Parthood". Applied Ontology 1 (2), 147-155. 
Wimsatt, William C. 1994: "The Ontology of Complex Systems: Levels of Organization, Perspectives, and Causal Thickets". Canadian Journal of Philosophy 24 (1), 207-274.

Winston, Morton, Robert Chaffin \& Douglas Herrmann 1987: "A Taxonomy of Part-Whole Relations". Cognitive Science 11 (4), 417-444.

Winther, Rasmus G. 2011: "Part-whole Science". Synthese 178 (3), 397-427. 